\title{
Metastatic castration-resistant prostate cancer in very elderly patients: challenges and solutions
}

\author{
This article was published in the following Dove Press journal: \\ Clinical Interventions in Aging \\ 22 December 2016 \\ Number of times this article has been viewed
}

\author{
Orazio Caffo \\ Francesca Maines \\ Mimma Rizzo \\ Stefania Kinspergher \\ Antonello Veccia \\ Medical Oncology Department, Santa \\ Chiara Hospital, Trento, Italy
}

\begin{abstract}
The treatment of elderly patients with cancer is usually viewed by clinicians as a challenge, because of the age-related decline in normal organ function and the frequent concomitant administration of multiple drugs for comorbid conditions. Clinicians therefore tend not to prescribe antineoplastic agents (mainly in the case of chemotherapy) to elderly patients, with the fear of excess toxicity leading to an unfavorable cost:benefit ratio. The cutoff age defining a cancer patient as elderly is usually 70 years, but over the last 10 years clinicians have paid more attention to functional status, as evaluated by means of a comprehensive geriatric assessment and comorbidity burden, rather than chronological age. In the case of metastatic castration-resistant prostate cancer (mCRPC), depending on their age at the time of diagnosis of PC, many (if not most) of the patients are more than 70 years old, and a fair number are very elderly patients aged $\geq 80$ years. The availability of various agents capable of significantly prolonging survival has dramatically changed the therapeutic landscape of mCRPC patients, but very elderly patients are usually underrepresented in pivotal trials. This narrative review considers the available data concerning elderly and very elderly mCRPC patients enrolled in pivotal trials and the information provided by reports of everyday clinical practice, in order to explore the challenges related to the clinical management of this special population.
\end{abstract}

Keywords: prostate cancer, elderly, castration-resistant

\section{Introduction}

The prostate cancer (PC) scenario has changed over the last few years, particularly in terms of the management of metastatic castration-resistant PC (mCRPC), and the number of elderly patients with $\mathrm{MCRPC}$ will significantly increase in the near future as a result of the global increase in life expectancy and the availability of life-prolonging treatments. ${ }^{1,2}$

Old men are usually defined as those aged $>65$ years, but chronological age alone is not very informative for clinical decision making. Since the 1990 s, there has been an increase in the use of such terms as "frailty" and "biological age", thus indicating that clinicians prefer to classify patients on the basis of their clinical status, functional reserve, and vulnerability. There are important differences between elderly and younger subjects that may affect treatment tolerance: the decline in normal organ function can lead to different drug metabolism and clearance; age-related physiological changes, such as a relative increase in body fat, reduced water content, and reduced muscle mass, influence drug distribution; elderly patients are more likely to be taking multiple drugs for comorbid conditions that may interact with cancer treatments; and elderly patients looking back on a fulfilled life may have a different perception of a cancer diagnosis and the side effects of cancer treatment from that of their younger counterparts. ${ }^{3}$
Correspondence: Orazio Caffo Medical Oncology Department, Santa Chiara Hospital, 9 Largo Medaglie d'Oro,

Trento 38100 , Italy

Tel +39046I 902478

Fax $+39046 \mid 903364$

Email orazio.caffo@apss.tn.it 
The guidelines for the treatment of older men with PC published by the International Society of Geriatric Oncology (SIOG) emphasize the importance of carefully assessing comorbidities and health status in the elderly. ${ }^{4} \mathrm{~A}$ complete geriatric assessment may also help to characterize the general health of elderly patients more precisely and make it easier to decide on the most appropriate treatment for individual patients by balancing considerations concerning relative life expectancy with the aggressiveness of cancer.

There is a lack of clinical trial data concerning the elderly, because trial-exclusion criteria often include comorbidities, reduced performance status (PS), concomitant medications, and impaired functional organ capacity, all of which are typical of advanced age, and because clinicians are concerned about the toxic effects of the trial treatment. Consequently, elderly patients recruited in clinical trials do not represent the elderly general population, and caution is required when trial results are generalized to everyday practice. ${ }^{5}$

Age-stratified, retrospective analyses of MCRPC populations have confirmed the survival benefit of cytotoxic and new hormonal agents in both older and younger patients, although the former were characterized by increased toxicity rates and dose reductions. ${ }^{6-9}$ The cutoff age discriminating younger and older patients in these analyses is usually 75 years, but looking at the median age of the enrolled patients, it is clear that a fair number are $>80$ years old; furthermore, octogenarians with $\mathrm{mCRPC}$ are frequently seen in everyday clinical practice. This narrative review describes the available treatments for mCRPC in elderly subjects, paying particular attention to the benefit:harm ratio in octogenarians.

\section{Older patients with cancer}

Aging is physiologically associated with a progressive reduction in organ function and an increased susceptibility to diseases: it is characterized by higher incidence of cardiovascular diseases, a decline in lung function (less compliance of the chest wall, weaker respiratory muscles, and a poor response to hypoxia and hypercapnia), impaired renal function (decreased glomerular filtration rate and consequent hypertension), reduced liver function, and a smaller bone marrow reserve. ${ }^{10-12}$

At the same time, advanced age is a risk factor for the development of cancer because of longer exposure to carcinogens, the greater susceptibility of older tissues to environmental carcinogens, and changes in the bodily environment (chronic inflammation and increased insulin resistance). ${ }^{13}$ A higher incidence of tumors in the elderly has recently been confirmed by the National Cancer Institute's Surveillance, Epidemiology and End Results database. ${ }^{14}$
It has been suggested that most cancers are diagnosed at a more advanced stage in older people. ${ }^{13}$ In the case of PC, the UCSF Cancer of the Prostate Strategic Urologic Research Endeavor database has confirmed that older patients are more likely to have high-risk PC at the time of diagnosis and less likely to receive local therapy..$^{15}$ It has also been reported that men aged 70-75 years have higher pathological Gleason scores, larger tumor volumes, and more advanced disease after prostatectomy than younger men. ${ }^{16}$

The definition of elderly patients usually refers to chronological age, although no unanimous cutoff age has been established. In clinical studies, the maximum age for enrolment is usually 70 years, but this does not reflect the real prevalence of the elderly in the general population. According to the National Comprehensive Cancer Network ( $\mathrm{NCCN}$ ) guidelines, older patients should be classified into three categories: young old (65-75 years), old (76-85 years), and oldest old patients $\left(>85\right.$ years). ${ }^{17}$

Various aspects need to be considered in decision-making processes involving elderly patients. The most important factor to take into account is life expectancy, particularly when tumors progress slowly or adjuvant treatment is indicated. ${ }^{12}$ It is also useful to define patient frailty, a dynamic heterogeneous and multidimensional process in which disability, geriatric syndromes, comorbidities, cancer, and age may alter a steady state and make a patient more vulnerable to diseases. ${ }^{11}$ A fit older patient may receive the same treatments as younger patients, whereas a frail patient may be more exposed to therapy-related toxicities and an uncertain benefit in terms of response and survival. ${ }^{12,18}$ Finally, age may also influence the choice of the type of treatment. Elderly patients with cancer are more exposed to the early and late complications of surgery, and at higher risk of a longer hospital stay. Moreover, although local treatments, such as radiotherapy, radiofrequency ablation, or endoscopic tumor ablation, may be well tolerated, systemic therapies may have a different impact on a patient's quality of life and response outcomes, eg, the hormonal therapies used in breast cancer and PC are usually well tolerated and have a favorable benefit:risk ratio, whereas chemotherapy is associated with a higher incidence of drug-related adverse events (AEs), and targeted therapies may cause severe cardiovascular and hematological toxicities. ${ }^{13}$

Unfortunately, very little evidence was available to guide the choice of treatment until the SIOG established a panel of experts to develop recommendations concerning the geriatric assessment of cancer patients. After reviewing the literature, and despite the paucity of data from randomized studies, the 
panel stated that a geriatric assessment could be valuable in oncology practice as a means of detecting impairments that are not identified during routine history-taking and physical examinations, predicting severe treatment-related toxicity and survival, and guiding the choice of treatment and its intensity. It also suggested a series of instruments (scales and tests) capable of assessing patients' health on the basis of their functional status, comorbidities, nutritional status, polypharmacy status, cognitive function, psychological status, socioeconomic issues, and geriatric syndromes (see Table 1). ${ }^{19}$

The Comprehensive Geriatric Assessment (CGA) is currently considered the backbone of evaluations of the health of elderly cancer patients in most oncology guidelines. ${ }^{17,20}$ The results of the CGA should be documented in a patient's clinical records, so that they are available at the time of treatment decision making. It was also suggested that a two-step

Table I Comprehensive geriatric assessment domains and main means of evaluation

\begin{tabular}{|c|c|}
\hline Domain & Evaluation \\
\hline \multirow[t]{3}{*}{ Functional status } & Performance status \\
\hline & Activities of daily living (ADL) \\
\hline & Instrumental ADL (IADL) \\
\hline \multirow[t]{6}{*}{ Comorbidities } & Cumulative Illness Rating Scale for \\
\hline & Geriatrics (CIRS-G) \\
\hline & Charlson Comorbidity Index $(\mathrm{CCl})$ \\
\hline & Adult Comorbidity Evaluation 27 (ACE-27) \\
\hline & OARS Multidimensional Functional \\
\hline & Assessment Questionnaire \\
\hline \multirow[t]{6}{*}{ Polypharmacy status } & Beers criteria \\
\hline & Medication Appropriateness Index (MAl) \\
\hline & Screening Tool of Older Persons' \\
\hline & Prescriptions (STOPP) criteria \\
\hline & Screening Tool to Alert Doctors to Right \\
\hline & Treatment (START) criteria \\
\hline \multirow[t]{2}{*}{ Nutritional status } & Mini-Nutritional Assessment (MNA) \\
\hline & Body mass index (BMI) \\
\hline \multirow[t]{4}{*}{ Cognitive function } & Mini-Mental State Examination (MMSE) \\
\hline & Montreal Cognitive Assessment (MoCA) \\
\hline & Blessed Orientation-Memory- \\
\hline & Concentration (BOMC) test \\
\hline Socioeconomic & Living conditions \\
\hline issues & Caregiver \\
\hline \multicolumn{2}{|l|}{ Psychological status } \\
\hline - Depression & Geriatric Depression Scale (GDS) \\
\hline - Distress & Distress Thermometer (DT) \\
\hline \multicolumn{2}{|l|}{ Geriatric syndromes } \\
\hline - Delirium & Confusion Assessment Method (CAM) \\
\hline - Frailty & Fried frailty criteria, Balducci frailty criteria \\
\hline - Fatigue & $\begin{array}{l}\text { Screening questionnaire to rate the } \\
\text { severity of fatigue }\end{array}$ \\
\hline - Falls & Multifactorial risk assessment \\
\hline - Osteoporosis & Fracture risk assessment \\
\hline
\end{tabular}

Abbreviation: OARS, Older Americans Resources and Services. approach should be adopted by initially assessing frailty using such instruments as the Vulnerable Elders Survey 13 or G8 to identify patients who may benefit from the CGA. ${ }^{17,20}$

\section{Guidelines for the management of older patients with prostate cancer}

The SIOG developed specific guidelines for the management of localized and advanced PC in men aged $>70$ years in $2010,{ }^{21}$ and these were updated by a new multidisciplinary SIOG working group in 2014. ${ }^{4}$ According to the SIOG guidelines, decision making for patients with PC should include three steps: an initial screening phase using the G8 scale, followed by the assessment of comorbidities (Cumulative Illness Rating Scale for Geriatrics [CIRS-G]), dependence status (activities of daily living [ADL] and instrumental ADL [IADL] scales), nutritional status (weightloss estimation), and screening for neuropsychological problems, and finally verification of the reversibility of any impairments. Older patients with PC can be divided into three groups: fit, vulnerable, and frail. Fit patients are those with a G8 score $>14$ who can tolerate any form of standard treatment. Vulnerable patients are those with a G8 score $\leq 14$ who could receive standard treatment after specific geriatric interventions to treat any of the following reversible impairments: grade 2 comorbidities, one grade 3 comorbidity, one or two ADL impairments (apart from incontinence), risk of malnutrition reversible through geriatric intervention, and depression that can be controlled by medical treatment. Frail patients are those with a G8 score $\leq 14$ who can receive an adapted cancer treatment after geriatric intervention(s) to treat one or more of the following irreversible impairments: several grade 3 comorbidities or one grade 4 comorbidity, more than two ADL abnormalities, severe malnutrition, abnormal IADL, or neuropsychological problems. ${ }^{4}$

The SIOG working group also evaluated the standard treatments for both localized and advanced PC: it was observed that the elderly patients included in the analyzed studies were fit or in unspecified health and received a clinical benefit that was similar to that of younger adult patients. The SIOG guidelines recommend defining treatment on the basis of the patient's preferences and health status rather than chronological age. ${ }^{4}$

In cases of localized disease, fit and vulnerable patients at high risk according to D'Amico classification and with a life expectancy of at least 10 years should receive standard treatment with curative intent (radical prostatectomy, external beam radiotherapy, or brachytherapy). Patients at low-to-intermediate risk could be included in surveillance 
or watchful-waiting programs. Caution is necessary when androgen-deprivation therapy (ADT) is used to treat nonmetastatic disease, because it may lead to a nonsignificant benefit in terms of survival but may also increase the risk of cardiovascular diseases, osteoporosis, fractures, and diabetes., ${ }^{4,22}$

In cases of advanced disease, ADT by means of surgical castration or luteinizing hormone-releasing hormone (LHRH) agonists or antagonists is the first-line treatment for hormone-sensitive cancer. Second-line treatment is the cessation of antiandrogen therapy, if given in combination with LHRH analogs. No hormonal therapy has shown a survival benefit from the second line onward. Given the high risk of osteoporosis and fractures in older men receiving ADT, they should receive calcium and vitamin D supplementation after assessing baseline bone mineral density.

When metastatic disease becomes castration-resistant, both new hormonal therapies (abiraterone acetate [AA] and enzalutamide [ENZ]) and chemotherapy can be offered. The new hormonal treatments are well tolerated and particularly suitable for asymptomatic or mildly symptomatic patients without visceral metastases, and survival outcomes in elderly patients are similar to those of younger patients.

Chemotherapy is also a valid option, particularly for more symptomatic patients and/or those with visceral disease: the data suggest that older age does not contraindicate chemotherapy. On the basis of the data coming from the TAX 327 study $^{23}$ and a retrospective analysis of patients aged $\geq 75$ years, ${ }^{24}$ 3-weekly docetaxel regimens can be offered to both fit and vulnerable older patients, whereas a weekly or 2-weekly docetaxel regimen should be considered in the case of frail patients.

Cabazitaxel or hormonal drugs (AA and ENZ) can be used in second-line treatment. Data from the TROPIC study showed that the survival advantage offered by cabazitaxel was independent of age, although primary prophylaxis with granulocyte colony-stimulating factor (G-CSF) is strongly suggested because of the high risk of febrile neutropenia in the elderly. ${ }^{4,25}$ The best sequence of these drugs in elderly patients is a subject of research.

Regardless of frailty, palliative treatments are used to control pain and other symptoms, and include radiotherapy, radiopharmaceuticals, bone-targeted therapies, surgery, and medical treatments. The guidelines do not recommend routine PSA screening in men aged $\geq 70$ years or with a life expectancy $\leq 10$ years; however, it is reasonable that the decision is based on health status rather than age. ${ }^{4}$

In addition to the guidelines developed by the SIOG, other guidelines have been proposed in order to optimize the management of elderly patients with PC. The guidelines proposed by the American Urological Association and the European Organisation for Research and Treatment of Cancer do not consider comorbidities when evaluating treatment, whereas the NCCN and European Association of Urology (EAU) guidelines include estimates of risk and life expectancy. The EAU guidelines offer a specific section dedicated to the management of $\mathrm{PC}$ in older patients, and recognize the importance of CIRS-G and G8 in assessing comorbidities and health status. ${ }^{16}$

\section{Outcomes in older $\mathrm{MCRPC}$ patients treated with new agents}

Until 2004, mitoxantrone was the only approved drug for the treatment of $\mathrm{mCRPC}$, although this agent was rarely proposed because its palliative effect had no impact on survival outcomes. Subsequently, it was found that a number of other agents significantly prolonged the overall survival (OS) of mCRPC patients (Table 2), and the life expectancy of patients who have become castration-resistant is now longer than at the beginning of this century, because of the possibility of using these new drugs sequentially.

Table 2 Pivotal trials of new agents in mCRPC

\begin{tabular}{|c|c|c|c|c|c|c|c|c|}
\hline Trial & Drug & References & $\begin{array}{l}\text { Treatment } \\
\text { line }\end{array}$ & $\begin{array}{l}\text { Control } \\
\text { arm }\end{array}$ & Primary end point & HR & $\begin{array}{l}\text { Median } \\
\text { age (years) }\end{array}$ & $\begin{array}{l}\text { Patients } \\
\geq 75 \text { years old }\end{array}$ \\
\hline TAX 327 & Docetaxel & 23 & First & Mitoxantrone & Overall survival & 0.76 & 68 & $20 \%$ \\
\hline TROPIC & Cabazitaxel & 31 & Second & Mitoxantrone & Overall survival & 0.7 & 67 & $19 \%$ \\
\hline COU-AA-30I & Abiraterone & 36 & Second & Prednisone & Overall survival & 0.8 & 69 & $28 \%$ \\
\hline AFFIRM & Enzalutamide & 41 & Second & Placebo & Overall survival & 0.63 & 69 & $25 \%$ \\
\hline \multirow[t]{2}{*}{ COU-AA-302 } & Abiraterone & 38,39 & First & Prednisone & Overall survival & 0.81 & 71 & $34 \%$ \\
\hline & & & & & Radiographic-free survival & 0.45 & & \\
\hline \multirow[t]{2}{*}{ PREVAIL } & Enzalutamide & 42 & First & Placebo & Overall survival & 0.71 & 72 & $36 \%$ \\
\hline & & & & & Radiographic-free survival & 0.19 & & \\
\hline ALSYMPCA & Radium-223 & 44 & First/second & Placebo & Overall survival & 0.7 & 71 & $28 \%$ \\
\hline IMPACT & Sipuleucel-T & 46 & First & Placebo & Overall survival & 0.78 & 72 & NR \\
\hline
\end{tabular}

Abbreviations: mCRPC, metastatic castration-resistant prostate cancer; HR, hazard ratio; NR, not reported. 


\section{Docetaxel}

In 2004, it was found that docetaxel lead to significant advantages over mitoxantrone in terms of symptom control and survival. ${ }^{23}$ The Phase III TAX 327 trial randomized 1,006 mCRPC patients to 3-weekly treatment with docetaxel + prednisone $(\mathrm{PDN})$ or weekly treatment with docetaxel + PDN or mitoxantrone + PDN. In comparison with mitoxantrone + PDN, 3-weekly docetaxel + PDN improved median survival (18.9 vs 16.5 months, $P=0.009$ ), increased the proportion of patients with $\geq 50 \%$ reduction in prostate-specific antigen (PSA) levels (45\% vs $32 \%, P<0.001)$, reduced pain $(35 \%$ vs $22 \%, P=0.01$ ), and improved quality of life ( $22 \%$ vs $13 \%$, $P=0.009)$. The survival advantage was confirmed after a 4-year extended follow-up (19.2 vs 16.3 months, $P=0.004){ }^{26}$ The main grade 3-4 toxicity was neutropenia (32\%). On the basis of these findings, docetaxel became the treatment of choice for patients with mCRPC.

The tolerability of docetaxel remains an important issue in everyday clinical practice: clinicians are concerned about the tolerability of chemotherapy in elderly patients, and often prefer weekly docetaxel over the standard 3-weekly regimen because of its more manageable safety profile, even though the TAX 327 trial did not demonstrate a survival advantage over mitoxantrone in the weekly arm. ${ }^{23}$

A recent retrospective analysis of TAX 327 has shown that 3-weekly docetaxel remains the standard of care for fit elderly patients, because of its better survival outcomes and the absence of any significant differences in the tolerability of the weekly or 3-weekly regimen in older men. ${ }^{6}$ However, as pointed out by the authors, the selection of the patients enrolled in Phase III clinical trials, the small proportion of enrolled patients aged $>75$ years $(20 \%)$, and the retrospective nature of the subgroup analyses mean that caution is required when translating the results to everyday clinical practice.

There have been some recently published reports concerning the management of $\mathrm{MCRPC}$ patients in clinical practice. A pooled analysis of two Phase II clinical trials of weekly docetaxel in CRPC patients did not find any significant differences in efficacy or toxicity between patients aged $>70$ years and younger patients. ${ }^{27}$ Italiano et al reviewed the clinical practice use of docetaxel-based chemotherapy in 175 mCRPC elderly patients using a cutoff age of 75 years and obtained similar results: ${ }^{24} 54 \%$ of the patients were treated using a standard 3-week regimen and $46 \%$ received weekly schedules, and the authors found that the patients receiving weekly docetaxel were older ( $>80$ years) and had a poorer PS ( $\geq 2)$ than those treated with the standard regimen. Their conclusions were that docetaxel can be safely administered to elderly patients with a good PS, and that age by itself should not be used as a criterion to deny mCRPC patients a potentially effective treatment.

A recent study of mCRPC patients aged $\geq 80$ years, ${ }^{28}$ of whom only $32.2 \%$ were treated with the standard 3-week regimen, found that the treatment was well tolerated, with a low rate of grade 3-4 hematological and nonhematological toxicities. There was a statistically significant difference in OS in favor of patients with low CIRS-G scores, and OS was also better in patients with low ADL and IADL scores, although the difference was of only borderline statistical significance.

Using the same cutoff age of 80 years to define elderly, Leibowitz-Amit et al found no age-related differences in terms of PSA response rates or OS, although febrile neutropenia was more frequent in older than in younger patients. ${ }^{29}$ Similarly, an analysis of a small series of octogenarians, most of whom were treated with 3-weekly docetaxel, found significant response rates and grade 3-4 hematological AEs in $45 \%$ of the patients. ${ }^{30}$

\section{Cabazitaxel}

The randomized Phase III TROPIC trial compared the efficacy and safety of cabazitaxel $\left(25 \mathrm{mg} / \mathrm{m}^{2}\right.$ every 3 weeks $)+$ PDN (10 mg daily) with mitoxantrone $\left(12 \mathrm{mg} / \mathrm{m}^{2}\right.$ every 3 weeks) + PDN (10 mg daily) in $755 \mathrm{mCRPC}$ patients with progressive disease after docetaxel-based chemotherapy. ${ }^{31}$ Cabazitaxel was associated with longer median OS (15.1 vs 12.7 months, hazard ratio $[\mathrm{HR}]=0.7,95 \%$ confidence interval $[\mathrm{CI}]: 0.59-0.83 ; P<0.0001)$, and its other clinical benefits included increased progression-free survival (PFS), maintained PS, improved tumor and PSA responses, and longer time to tumor and PSA progression. Grade 3-4 AEs that were more frequent with cabazitaxel included neutropenia ( $82 \%$ vs $58 \%$ ), febrile neutropenia ( $8 \%$ vs $1 \%$ ), and diarrhea ( $6 \%$ vs $<1 \%$ ), but unlike treatment with docetaxel, there was no evidence of grade $\geq 3$ peripheral neuropathy.

The TROPIC trial included 139 patients aged $>75$ years: $69(18 \%)$ in the cabazitaxel group and $70(19 \%)$ in the mitoxantrone group. In these patients, the $\mathrm{AE}$ rates were greater than in the younger population in terms of diarrhea and neutropenia (55.7\% and $24.2 \%$ vs $44.5 \%$ and $17.6 \%$ ). No differences in neutropenia and diarrhea were found in subgroups classified by race, baseline liver or renal function, Eastern Cooperative Oncology Group (ECOG) PS, or previous chemotherapy. It is thus necessary to monitor bone marrow function carefully and consider primary prophylaxis with G-CSF in high-risk patients (age $>65$ years, 
poor PS, previous episodes of febrile neutropenia, extensive previous radiation, poor nutritional status, or other serious comorbidities).

A number of early access programs (EAPs) involving $6.2 \%-26.8 \%$ of patients aged $\geq 75$ years have demonstrated that cabazitaxel is well tolerated in a real-world setting. ${ }^{32-35}$ In an Italian EAP, G-CSF was more frequently used in older patients ( $66.8 \%$ vs $58.0 \%$ ), but the hematological tolerability of cabazitaxel seemed to be similar regardless of age. ${ }^{34}$

Additional data concerning a large elderly population treated with cabazitaxel was provided by cumulative reports from European EAPs that stratified 746 men on the basis of their age (421 aged $<70,180$ aged 70-74, and 145 aged $\geq 75$ years). ${ }^{8}$ The number of cabazitaxel cycles, dose reductions for any cause, dose delays possibly related to cabazitaxel AEs, and tolerability were similar in the three age-groups, but prophylactic G-CSF was more frequently used by men aged $\geq 70$ years. Multivariate analysis showed that age $\geq 75$ years was a predictor of grade $\geq 3$ neutropenia and/or neutropenic complications (febrile neutropenia and neutropenic sepsis) together with the first cabazitaxel cycle, no G-CSF prophylaxis at a given cycle, a neutrophil count of $<4,000 / \mathrm{mm}^{3}$ before cabazitaxel injection, and fewer than ten previous cycles of docetaxel. The authors concluded that prophylactic use of G-CSF, particularly at the time of cycle 1 and in men aged $\geq 75$ years, improved tolerability in elderly patients treated with cabazitaxel.

\section{Abiraterone}

AA has proved to be efficacious as both first- and secondline treatment, and significantly improves the OS of mCRPC patients. Grade 3-4 toxicities of interest described in patients treated with AA have been fluid retention and edema (2\%), hypokalemia $(2 \%-3 \%)$, cardiac disorders $(4 \%-6 \%)$, liver-function test abnormalities $(3 \%-5 \%)$, and hypertension $(1 \%-4 \%)$. The pivotal COU-AA-301 trial tested the drug in patients who had previously received docetaxel, and compared AA + PDN with placebo + PDN: the final analysis showed that median OS was longer in the AA group (15.8 vs 11.2 months, $\mathrm{HR}=0.74) .{ }^{36}$

A post hoc analysis of the COU-AA-301 trial by Mulders et al in which "elderly" was defined as age $>75$ years showed that AA improved clinical outcomes, including OS, and was well tolerated in both elderly and younger patients, and that the drug's safety profile and survival benefit in comparison with PDN in the elderly patients were similar to those recorded in the overall patient population. ${ }^{7}$ In the elderly subgroup treated with AA, the authors observed a slightly higher incidence of cardiac disorders (particularly atrial fibrillation and tachycardia) than in those receiving PDN, but without a significant increase in treatment interruptions, treatment discontinuations, or incidence of AEs leading to death; moreover, the older patients had similar treatment duration to the younger population (36 vs 32 months).

The overall efficacy data were similar to those observed in a group of $47 \mathrm{mCRPC}$ patients aged $>80$ years who were treated in the Italian AA "named patient" program after docetaxel failure outside a randomized clinical trial. ${ }^{37}$ This analysis retrospectively confirmed that the treatment was efficacious and safe in this age-group and did not significantly increase the risk of AEs, and that response rates and survival outcomes were similar to those observed in patients aged $<80$ years.

The COU-AA-302 trial compared AA + PDN with placebo + PDN in a first-line setting: the results published at the time of second interim analysis only met the planned coprimary end point of radiological PFS and not that of OS, but the differences between the two arms were already statistically significant. ${ }^{38}$ The final results showed significantly longer median OS in the AA + PDN group (34.7 vs 30.3 months), with an HR of 0.81 meeting the predefined efficacy criterion. ${ }^{39}$

The efficacy and safety of AA in elderly chemonaive patients has been evaluated in a post hoc analysis of 350 patients aged $>75$ years enrolled in the COU-AA-302 trial, ${ }^{40}$ in which the percentage of patients with an ECOG PS of 1 was higher among older than younger patients. Like those aged $<75$ years, the elderly patients treated with AA experienced significant improvements in radiographic PFS, OS, and all of the secondary end points (time to PSA progression, time to the start of chemotherapy, time to the use of opiates for cancer-related pain, and time to the deterioration of ECOG PS) in comparison with patients treated with PDN alone. Subsequent docetaxel therapy was more frequent among younger than elderly patients. Dose reductions and treatment interruptions were infrequent in both age-groups. However, a higher rate of AA discontinuations due to AEs was observed in elderly patients than in those aged $<75$ years ( $15 \%$ vs $5 \%$ ). The safety profile was similar in both groups, with a higher incidence of grade 3-4 AEs (57\% vs 40\%) and peripheral edema.

These findings are comparable with those of LeibowitzAmit et al, who recently retrospectively compared the efficacy and tolerability of AA in 34 patients aged $>80$ years (median age $=85$ years) and 328 patients aged $<80$ years who received AA outside a clinical trial. They did not find 
any significant difference in PSA response rate, PFS, OS, or AA-related AEs between the two groups. ${ }^{29}$ There was a nonsignificant trend to more frequent toxicity-related drug discontinuations among the octogenarians (9\% vs 7\%).

\section{Enzalutamide}

Like AA, ENZ has been tested in the first- and second-line treatment of mCRPC, and leads to a significant survival advantage. Grade 3-4 toxicities of interest observed in pivotal trials were hypertension $(7 \%)$, cardiac events $(1 \%-3 \%)$, and seizures $(<1 \%)$. However, potential interactions with some drugs have been described, because ENZ is a potent inducer of CYP3A4 and a moderate inducer of CYP2C9 and CYP2C19 in vivo. The AFFIRM trial compared ENZ with placebo in patients previously treated with docetaxel, and found that median OS was 18.4 months in the ENZ group and 13.6 months in the placebo group $(\mathrm{HR}=0.63) .{ }^{41}$

The published results of a post hoc subgroup analysis of the AFFIRM trial revealed comparable clinical outcomes and toxicities in $\mathrm{MCRPC}$ patients aged $<75$ years and $>75$ years treated with ENZ after docetaxel failure, and showed that the tolerability of the drug and its clinically significant benefit in elderly patients were consistent with those observed in the pivotal clinical trial as a whole. ${ }^{9}$

The placebo-controlled Phase III PREVAIL trial of ENZ in a first-line setting reproduced the design of the COU-AA302 trial, and had the same coprimary end points, both of which were met at the time of the planned interim analysis: the ENZ group showed better median radiographic PFS (not reached vs 3.9 months) and better median OS (32.4 vs 30.2 months). ${ }^{42}$

Graff et al reported a planned subgroup analysis of chemonaïve ENZ-treated patients aged $\geq 75$ years treated in the PREVAIL trial. ${ }^{43}$ A total of 609 patients aged $\geq 75$ years $(35 \%)$ were enrolled (median age $=80$ years, range: 75-93 years), of whom 307 received ENZ and 292 placebo. As expected, the patients in the elderly group had worse PS than those aged $<75$ years, and a higher percentage had cardiovascular disease at baseline $(26.9 \%$ vs $16.5 \%$ ). They also had longer median time from diagnosis to trial participation (88.3 vs 53.4 months), heavier disease burden, and higher median PSA levels at baseline ( $73.3 \mathrm{vs} 37.3 \mathrm{ng} / \mathrm{mL}$ ). The authors reported similar improvements in OS and radiographic PFS in both age-groups, with comparable median duration of treatment. The elderly patients in either treatment group had a higher incidence of any grade $>3 \mathrm{AE}$, falls, fractures, decreased appetite, and asthenia than patients aged $<75$ years, and the AE rate was higher in the elderly in the ENZ arm than those in the placebo arm, but only the incidence of falls remained higher in the ENZ-treated patients after adjusting for the length of treatment exposure. The authors concluded that ENZ is safe and well tolerated in the elderly. As in the COU-AA-301 trial, a lower percentage of elderly patients received subsequent antineoplastic therapy in terms of chemotherapy (eg, docetaxel $32.3 \%$ vs $51.3 \%$ or cabazitaxel $3.3 \%$ vs $12.7 \%$ ) or hormonal strategies (eg, AA $27.6 \%$ vs $35.7 \%$ ). The most frequent cause of death of the elderly in both arms was the progression of PC and not age-related comorbidities.

\section{Radium-223}

Radium-223 is an $\alpha$-emitter that acts as a bone-seeking agent and has a good hematological toxicity profile (grade 3-4 thrombocytopenia $6 \%$, neutropenia 3\%). It was tested in the pivotal ALSYMPCA trial, which randomized 921 mCRPC patients with symptomatic bone metastases but without visceral or large $(>3 \mathrm{~cm})$ nodal metastases to receive radium-223 or placebo. ${ }^{44}$ The patients may have been chemonaïve (if they had refused or been ineligible for docetaxel) or pretreated with docetaxel. The results showed a significant improvement in OS (median: 14.9 vs 11.3 months, $\mathrm{HR}=0.7,95 \% \mathrm{CI}: 0.58-0.83 ; P<0.001)$.

All of the other secondary efficacy end points significantly favored treatment with radium-223, and it was confirmed that the survival advantage did not depend on previous docetaxel use. $^{45}$ The study population had a median age of 71 years, with $28 \%$ of the patients being $\geq 75$ years old. No analysis of the pivotal trial or EAP has specifically investigated the clinical outcomes and safety profile of radium-223 in the elderly. However, as radium-223 treatment is characterized by low rates of hematological and nonhematological toxicities, it can be postulated that age does not affect its tolerability or efficacy.

\section{Sipuleucel-T}

Sipuleucel-T is an autologous dendritic cell-based vaccine that significantly improves survival in asymptomatic patients with slowly evolving mCRPC. In the pivotal placebo-controlled IMPACT trial, median survival in the sipuleucel-T group was 25.8 months against 21.7 months in the placebo group $(\mathrm{HR}=0.78,95 \% \mathrm{CI}: 0.61-0.98$; $P=0.03) .{ }^{46}$ In this trial, sipuleucel-T was associated with low rates $(1 \%-3 \%)$ of grade $3-4$ toxicities (chills, fatigue, arthralgia, pain, and asthenia), and on the basis of these findings, the drug was approved by the US Food and Drug Administration (FDA) in 2010. However, the European 
Medicines Agency has recently withdrawn the vaccine's marketing authorization in the European Union, because the lack of evidence supporting the mechanism proposed by sipuleucel-T's manufacturer (which was underlined by the FDA reviewers) raised the concern that the survival benefit may have been due to a flaw in the trial design or the chance imbalance of unmeasured prognostic variables. Since the drug was approved by the FDA, unpublished data from internal FDA documents concerning the IMPACT trial have become available, and additional considerations concerning the efficacy of sipuleucel-T have been published. ${ }^{47}$ Surprisingly, it seems that the active treatment had no effect on survival in younger patients ( 29 vs 28.2 months, $\mathrm{HR}=1.41$, 95\% CI: $0.87-2.29)$, but it did in older patients (23.4 vs 17.3 months, $\mathrm{HR}=0.58,95 \% \mathrm{CI}: 0.43-0.76)$, thus suggesting that the overall results were driven entirely by this difference. However, there was also a great difference in median survival between the patients aged $<65$ years and those aged $\geq 65$ years in the placebo ( 28.2 vs 17.2 months), and as the number of reinfused cells was lower than that of the cells removed because of the way they were managed (planned removal, cold storage, no exposure to growth agents), it can be hypothesized that the older patients in the placebo arm were harmed more by the cell loss than their younger counterparts. Moreover, as the proposed mechanism of action of sipuleucel- $T$ is related to $\mathrm{T}$ cells, immunosenescence may affect its efficacy, although preliminary data from the Phase IV PROCEED study do not suggest any significant differences in terms of product parameters (total nucleated cell count, antigen-presenting cell count, and antigen-presenting cell activation) between the 250 patients aged $\geq 80$ years and the 1,004 patients aged $<80$ years. ${ }^{48}$

\section{Discussion}

Older cancer patients (usually defined as those aged $>70$ years) have always been a special population, and this was particularly true when chemotherapy was the only weapon in the hands of clinicians. The fear of excess toxicities because of comorbidities and an age-related reduction in physiological functions usually led to the elderly being excluded from or underrepresented in clinical trials. In order to overcome this limitation, special trials have been specifically designed for older patients with some types of cancers, such as non-smallcell lung cancer. ${ }^{49}$

In the case of $\mathrm{PC}$, problems due to age-related reduced compliance to treatment were only marginal for as long as ADT was the only therapeutic strategy, and there were survival-prolonging agents available once patients developed resistance to castration. However, this changed when it was found that docetaxel offered a survival advantage in mCRPC patients.

Although the toxicity profiles of the new-generation hormonal agents are different from those of chemotherapeutic agents, their use in the elderly should be cautiously considered. As PC typically affects the elderly, all of the pivotal trials of the new agents included a population whose median age ranged between 67 and 72 years (Table 2), and specific reports included a description of the characteristics of those aged $\geq 75$ years (Table 3), thus underlining the concept that this age-group represents a special population of elderly mCRPC patients.

However, this should be reconsidered, because octogenarians now represent a considerable proportion of the mCRPC patients encountered in everyday clinical practice. Moreover, as in the case of other tumors, the octogenarians enrolled in pivotal trials are quite different from those seen in daily practice, as they are highly selected, have fewer comorbidities, and their functional status is also likely to be better, which limits the generalization of the clinical trial results.

The availability of active agents with different toxicity profiles now makes it possible to adopt a personalized strategy based on the individual characteristics of each elderly patient, and so a CGA following the SIOG guidelines and an assessment of comorbidities play a central role. The first is crucial for identifying vulnerable and frail patients, who

Table 3 Pivotal trials of new agents in mCRPC: outcomes of patients $\geq 75$ years old

\begin{tabular}{|c|c|c|c|}
\hline Trial & References & Overall survival (months) & Relevant grade $3-4$ adverse events (\%) \\
\hline TAX 327 & 6 & 18.9 & Diarrhea (3), infection (9), fatigue (10), neutropenia (8) \\
\hline TROPIC & 31 & NR & Diarrhea $(55.7), *$ neutropenia $(24.2)^{* *}$ \\
\hline COU-AA-30I & 7 & 15.6 & Fatigue (I3), cardiac disorders (7), hypokalemia (6), hypertension (I) \\
\hline AFFIRM & 9 & 18.2 & Fatigue (9.5), edema (2.5) \\
\hline COU-AA-302 & 40 & 28.6 & $\begin{array}{l}\text { Fatigue (6), edema (I.I), diarrhea ( }(.1) \text {, cardiac disorders ( } 8.8) \text {, liver } \\
\text { toxicity ( } 8.2) \text {, hypertension ( } 4.4) \text {, hypokalemia }(4.4)\end{array}$ \\
\hline PREVAIL & 43 & 32.4 & $\begin{array}{l}\text { Fatigue (37.5),* diarrhea (I8.6),* hypertension (I I),* edema (I I I.4),* } \\
\text { cardiac disorders }(\mathrm{I} 3.2)^{*}\end{array}$ \\
\hline
\end{tabular}

Notes: *All grades; **all grades and patients $\geq 65$ years old.

Abbreviations: mCRPC, metastatic castration-resistant prostate cancer; NR, not reported. 
may receive standard treatment after medical intervention and adapted treatment, respectively, and the second defines the possible impact of comorbidities and their therapies on cancer-related treatment. Treatment decision making should be driven by a patient's physiological age and functional status, rather than their chronological age or PS alone, and appropriate patient selection allows clinicians to propose the treatment that best fits each patient at any specific time in their disease history, even after the age of 80 years.

In the case of chemotherapeutic agents, the main problem is the fear of excess toxicities, due mainly to age-related changes in the pharmacokinetics and pharmacodynamics of the administered drugs. ${ }^{50}$ Consequently, clinicians are reluctant to propose chemotherapy to even fit octogenarians, thus denying them a treatment that can significantly prolong their survival. On the other hand, although the new-generation hormonal agents are easy to administer and better tolerated, it is necessary to remember that they are not devoid of side effects and also require careful management.

However, these cautions should not exclude octogenarians from treatments that prolong their lives, and it has been reported that selected patients aged $>80$ years can significantly benefit from the sequential use of the new agents active on $\mathrm{mCRPC}$ in comparison with patients not receiving them. ${ }^{28}$

\section{Conclusion}

The cutoff age for defining elderly $\mathrm{mCRPC}$ patients should be moved to 80 years. In everyday clinical practice, this special population should be treated on the basis of the results of careful geriatric and comorbidity evaluation, and should be not excluded from new treatments simply because of their chronological age. Moreover, specific trials should be designed in order to extend the possibility for very elderly mCRPC patients to receive treatments that prolong their survival.

\section{Disclosure}

The authors report no conflicts of interest in this work.

\section{References}

1. Scosyrev E, Messing EM, Mohile S, Golijanin D, Wu G. Prostate cancer in the elderly: frequency of advanced disease at presentation and diseasespecific mortality. Cancer. 2012;118(12):3062-3070.

2. Ferlay J, Soerjomataram I, Dikshit R, et al. Cancer incidence and mortality worldwide: sources, methods and major patterns in GLOBOCAN 2012. Int J Cancer. 2015;136(5):E359-E386.

3. Handforth C, Clegg A, Young C, et al. The prevalence and outcomes of frailty in older cancer patients: a systematic review. Ann Oncol. 2015;26(6): 1091-1101.

4. Droz JP, Aapro M, Balducci L, et al. Management of prostate cancer in older patients: updated recommendations of a working group of the International Society of Geriatric Oncology. Lancet Oncol. 2014;15(9): e404-e414.
5. Yee KW, Pater JL, Pho L, Zee B, Siu LL. Enrollment of older patients in cancer treatment trials in Canada: why is age a barrier? J Clin Oncol. 2003;21(8):1618-1623.

6. Horgan AM, Seruga B, Pond GR, et al. Tolerability and efficacy of docetaxel in older men with metastatic castrate-resistant prostate cancer (mCRPC) in the TAX 327 trial. J Geriatr Oncol. 2014;5(2): 119-126.

7. Mulders PF, Molina A, Marberger M, et al. Efficacy and safety of abiraterone acetate in an elderly patient subgroup (aged 75 and older) with metastatic castration-resistant prostate cancer after docetaxel-based chemotherapy. Eur Urol. 2014;65(5):875-883.

8. Heidenreich A, Bracarda S, Mason M, et al. Safety of cabazitaxel in senior adults with metastatic castration-resistant prostate cancer: results of the European compassionate-use programme. Eur J Cancer. 2014;50(6):1090-1099.

9. Sternberg CN, de Bono JS, Chi KN, et al. Improved outcomes in elderly patients with metastatic castration-resistant prostate cancer treated with the androgen receptor inhibitor enzalutamide: results from the phase III AFFIRM trial. Ann Oncol. 2014;25(2):429-434.

10. Balducci L. Studying cancer treatment in the elderly patient population. Cancer Control. 2014;21(3):215-220.

11. Colloca G, Corsonello A, Marzetti E, et al. Treating cancer in older and oldest old patients. Curr Pharm Des. 2015;21(13):1699-1705.

12. Korc-Grodzicki B, Holmes HM, Shahrokni A. Geriatric assessment for oncologists. Cancer Biol Med. 2015;12(4):261-274.

13. Hoffe S, Balducci L. Cancer and age: general considerations. Clin Geriatr Med. 2012;28(1):1-18.

14. National Cancer Institute. Surveillance, Epidemiology, and End Results program. Available from: http://seer.cancer.gov. Accessed August 9, 2016.

15. Bechis SK, Carroll PR, Cooperberg MR. Impact of age at diagnosis on prostate cancer treatment and survival. J Clin Oncol. 2011;29(2): $235-241$.

16. Kalra S, Basourakos S, Abouassi A, et al. The implications of ageing and life expectancy in prostate cancer treatment. Nat Rev Urol. 2016;13(5): 289-295.

17. National Comprehensive Cancer Network [website on the Internet]. Available from: https://www.nccn.org. Accessed August 9, 2016.

18. Baijal P, Periyakoil V. Understanding frailty in cancer patients. Cancer J. 2014;20(5):358-366.

19. Wildiers H, Heeren P, Puts M, et al. International Society of Geriatric Oncology consensus on geriatric assessment in older patients with cancer. J Clin Oncol. 2014;32(24):2595-2603.

20. Italian Association of Medical Oncology [Associazione Italiana di Oncologia Medica]. [website on the Internet]. Available from: http:// www.aiom.it/2015. Accessed August 9, 2016.

21. Droz JP, Balducci L, Bolla M, et al. Management of prostate cancer in older men: recommendations of a working group of the International Society of Geriatric Oncology. BJU Int. 2010;106(4):462-469.

22. Kilari D, Dale W, Mohile SG. How we treat early systemic prostate cancer in older men. J Geriatr Oncol. 2014;5(4):337-342.

23. Tannock IF, de Wit R, Berry WR, et al. Docetaxel plus prednisone or mitoxantrone plus prednisone for advanced prostate cancer. $N$ Engl J Med. 2004;351(15):1502-1512.

24. Italiano A, Ortholan C, Oudard S, et al. Docetaxel-based chemotherapy in elderly patients (age 75 and older) with castration-resistant prostate cancer. Eur Urol. 2009;55(6):1368-1375.

25. Jha GG, Anand V, Soubra A, Konety BR. Challenges of managing elderly men with prostate cancer. Nat Rev Clin Oncol. 2014;11(6): 354-364.

26. Berthold DR, Pond GR, Soban F, de Wit R, Eisenberger M, Tannock IF. Docetaxel plus prednisone or mitoxantrone plus prednisone for advanced prostate cancer: updated survival in the TAX 327 study. J Clin Oncol. 2008;26(2):242-245.

27. Beer TM, Berry W, Wersinger EM, Bland LB. Weekly docetaxel in elderly patients with prostate cancer: efficacy and toxicity in patients at least 70 years of age compared with patients younger than 70 years. Clin Prostate Cancer. 2003;2(3):167-172. 
28. Veccia A, Caffo O, De Giorgi U, et al. Clinical outcomes in octogenarians treated with docetaxel as first-line chemotherapy for castration-resistant prostate cancer. Future Oncol. 2016;12(4):493-502.

29. Leibowitz-Amit R, Templeton AJ, Alibhai SM, et al. Efficacy and toxicity of abiraterone and docetaxel in octogenarians with metastatic castrationresistant prostate cancer. J Geriatr Oncol. 2015;6(1):23-28.

30. Wong HL, Lok SW, Wong S, Parente P, Rosenthal M. Docetaxel in very elderly men with metastatic castration-resistant prostate cancer. Prostate Int. 2015;3(2):42-46.

31. de Bono JS, Oudard S, Ozguroglu M, et al. Prednisone plus cabazitaxel or mitoxantrone for metastatic castration-resistant prostate cancer progressing after docetaxel treatment: a randomised open-label trial. Lancet. 2010;376(9747):1147-1154.

32. Wissing MD, van Oort IM, Gerritsen WR, et al. Cabazitaxel in patients with metastatic castration-resistant prostate cancer: results of a compassionate use program in the Netherlands. Clin Genitourin Cancer. 2013;11(3):238-250.

33. Heidenreich A, Scholz HJ, Rogenhofer S, et al. Cabazitaxel plus prednisone for metastatic castration-resistant prostate cancer progressing after docetaxel: results from the German compassionate-use programme. Eur Urol. 2013;63(6):977-982.

34. Bracarda S, Gernone A, Gasparro D, et al. Real-world cabazitaxel safety: the Italian early-access program in metastatic castration-resistant prostate cancer. Future Oncol. 2014;10(6):975-983.

35. Castellano D, Aparicio LM, Esteban E, et al. Cabazitaxel for metastatic castration-resistant prostate cancer: safety data from the Spanish expanded access program. Expert Opin Drug Saf. 2014;13(9): 1165-1173.

36. de Bono JS, Logothetis CJ, Molina A, et al. Abiraterone and increased survival in metastatic prostate cancer. $N$ Engl J Med. 2011; 364(21):1995-2005.

37. Maines F, Caffo O, De Giorgi U, et al. Safety and clinical outcomes of abiraterone acetate after docetaxel in octogenarians with metastatic castration-resistant prostate cancer: results of the Italian compassionate use named patient programme. Clin Genitourin Cancer. 2016;14(1):48-55

38. Ryan CJ, Smith MR, de Bono JS, et al. Abiraterone in metastatic prostate cancer without previous chemotherapy. $N$ Engl J Med. 2013; 368(2):138-148.

39. Ryan CJ, Smith MR, Fizazi K, et al. Abiraterone acetate plus prednisone versus placebo plus prednisone in chemotherapy-naive men with metastatic castration-resistant prostate cancer (COU-AA-302): final overall survival analysis of a randomised, double-blind, placebo-controlled phase 3 study. Lancet Oncol. 2015;16(2):152-160.
40. Smith MR, Rathkopf DE, Mulders PF, et al. Efficacy and safety of abiraterone acetate in elderly ( 75 years or older) chemotherapy naive patients with metastatic castration resistant prostate cancer. J Urol. 2015;194(5):1277-1284.

41. Scher HI, Fizazi K, Saad F, et al. Increased survival with enzalutamide in prostate cancer after chemotherapy. $N$ Engl J Med. 2012;367(13): 1187-1197.

42. Beer TM, Armstrong AJ, Rathkopf DE, et al. Enzalutamide in metastatic prostate cancer before chemotherapy. N Engl J Med. 2014;371(5): 424-433.

43. Graff JN, Baciarello G, Armstrong AJ, et al. Efficacy and safety of enzalutamide in patients 75 years or older with chemotherapy-naive metastatic castration-resistant prostate cancer: results from PREVAIL. Ann Oncol. 2016;27(2):286-294.

44. Parker C, Nilsson S, Heinrich D, et al. Alpha emitter radium-223 and survival in metastatic prostate cancer. $N$ Engl J Med. 2013; 369(3):213-223.

45. Hoskin P, Sartor O, O'Sullivan JM, et al. Efficacy and safety of radium223 dichloride in patients with castration-resistant prostate cancer and symptomatic bone metastases, with or without previous docetaxel use: a prespecified subgroup analysis from the randomised, double-blind, phase 3 ALSYMPCA trial. Lancet Oncol. 2014;15(12):1397-1406.

46. Kantoff PW, Higano CS, Shore ND, et al. Sipuleucel-T immunotherapy for castration-resistant prostate cancer. N Engl J Med. 2010; 363(5):411-422.

47. Huber ML, Haynes L, Parker C, Iversen P. Interdisciplinary critique of sipuleucel-T as immunotherapy in castration-resistant prostate cancer. J Natl Cancer Inst. 2012;104(4):273-279.

48. Nabhan C, Sartor O, Cooperberg MR, et al. Sipuleucel-T in metastatic castration-resistant prostate cancer (mCRPC) patients $\geq 80$ years-old: data from PROCEED. J Clin Oncol. 2014;32 Suppl 4:64.

49. Gridelli C, Perrone F, Gallo C, et al. Chemotherapy for elderly patients with advanced non-small-cell lung cancer: the Multicenter Italian Lung Cancer in the Elderly Study (MILES) phase III randomized trial. J Natl Cancer Inst. 2003;95(5):362-372.

50. Hurria A, Fleming MT, Baker SD, et al. Pharmacokinetics and toxicity of weekly docetaxel in older patients. Clin Cancer Res. 2006; 12(20 Pt 1):6100-6105.
Clinical Interventions in Aging

\section{Publish your work in this journal}

Clinical Interventions in Aging is an international, peer-reviewed journal focusing on evidence-based reports on the value or lack thereof of treatments intended to prevent or delay the onset of maladaptive correlates of aging in human beings. This journal is indexed on PubMed Central, MedLine,

\section{Dovepress}

CAS, Scopus and the Elsevier Bibliographic databases. The manuscript management system is completely online and includes a very quick and fair peer-review system, which is all easy to use. Visit http://www.dovepress. com/testimonials.php to read real quotes from published authors. 